\title{
A Double-Blind, Placebo-Controlled Trial Related to the Effects of Melatonin on Oxidative Stress and Inflammatory Parameters of Obese Women
}

Authors

Affiliations

\author{
N. Mesri Alamdari ${ }^{1}$, R. Mahdavi ${ }^{2}$, N. Roshanravan ${ }^{1}$, N. Lotfi Yaghin ${ }^{1}$, A. R. Ostadrahimi ${ }^{2}$, E. Faramarzi ${ }^{3}$
}

${ }^{1}$ Students Research Committee, School of Nutrition, Tabriz University of Medical Science, Tabriz, Iran ${ }^{2}$ Nutrition Research Center, School of Nutrition, Tabriz University of Medical Science, Tabriz, Iran

${ }^{3}$ Common Disease Risk Factors Management Institute, Tabriz University of Medical Sciences, Tabriz, Iran

\author{
Key words \\ obesity \\ - melatonin \\ - inflammation \\ oxidative stress \\ - low calorie diet
}

received 15.04.2014 accepted 30.06.2014

\section{Bibliography}

DOI http://dx.doi.org/

$10.1055 / \mathrm{s}-0034-1384587$

Published online:

August 15, 2014

Horm Metab Res 2015;

47: 504-508

(c) Georg Thieme Verlag KG Stuttgart · New York

ISSN 0018-5043

Correspondence

\section{R. Mahdavi}

Nutrition Research Center School of Nutrition

Tabriz University of Medical Science

Tabriz

Iran

Tel.: + 98/41/13357580

Fax: + 98/41/13357580

mahdavirez@hotmail.com

\section{Abstract}

$\checkmark$

Obesity, the global epidemic health problem, results in chronic disorders. Melatonin supplementation may prevent the adverse health consequences of obesity. The aim of this study was to assess the effects of melatonin supplementation on inflammatory and oxidative stress parameters in obese women. In randomized, double-blind, placebo-controlled trial, 44 obese women were randomly assigned to melatonin $(\mathrm{n}=22)$ and placebo $(n=22)$ groups. Subjects were supplemented with a daily dose of $6 \mathrm{mg}$ melatonin or placebo with low calorie diet for 40 days. Serum TNF- $\alpha$, IL-6, hsCRP, TAC, and MDA levels were assessed before and after intervention. In the melatonin group, mean serum TNF- $\alpha$, IL-6, hsCRP, and MDA levels decreased significantly

\section{Introduction}

$\nabla$

Obesity has become a global epidemic problem throughout the world. The total prevalence of obesity in the world is $10 \%$ in men and $14 \%$ in women. It is estimated that $13.6 \%$ of men and $29.5 \%$ of women are obese in Iran [1]. Increasing evidence shows that obesity is associated with inflammatory and oxidative stress responses, which cause chronic disturbances including cardiovascular disease, type 2 diabetes, dyslipidemia, cancers, and other metabolic and mental health disease [2-4].

Adipose tissue produces and releases a variety of proinflammatory cytokines including TNF- $\alpha$, IL-6, and adipokines leptin and adiponectin, which can induces the production of reactive oxygen species (ROS) and generate a process known as oxidative stress (OS) [5]. Therefore, obese subjects have higher levels of oxidative biomarkers [6]. Moreover, a growing body of evidence indicates that by increasing adipose $(\mathrm{p}<0.05)$ from $3.52 \pm 0.72 \mathrm{pg} / \mathrm{ml}, 27.12 \pm 6.32 \mathrm{pg} / \mathrm{ml}$, $2.54 \pm 0.49 \mathrm{mg} / \mathrm{l}$, and $3.81 \pm 0.29 \mathrm{nmol} / \mathrm{l}$ to $1.73 \pm 0.07$, $16.34 \pm 6.32,1.67 \pm 0.27$, and $2.79 \pm 0.29$, respectively. Whilst in the placebo group the decrease in values were not statistically significant. Mean TAC level increased slightly (from $1.11 \pm 0.30$ to $1.14 \pm 0.45 \mathrm{mmol} / \mathrm{l}$ ) in the melatonin group whereas it decreased slightly (from $1.13 \pm 0.15$ to $1.08 \pm 0.21 \mathrm{nmol} / \mathrm{l}$ ) in the placebo group. Significant differences were observed only for TNF- $\alpha$ $(\mathrm{p}=0.02)$ and IL-6 $(\mathrm{p}=0.03)$ between the 2 study groups. Considering the improvements in inflammatory and oxidative stress factors in obese women, it seems that melatonin supplementation may provide beneficial effects in obesity treatment by ameliorating some of its complications. However, further studies are needed to make concise conclusions. tissues, the activity of antioxidant enzymes and total antioxidant capacity diminishes significantly [7]. It seems more reasonable to consider the other means in addition to the current treatments for obesity such as dietary intervention, behavioral modification, and lifestyle changes, to prevent the adverse health consequences of obesity and related metabolic disorders $[8,9]$. New data have revealed the beneficial effects of melatonin as a nutritional supplement in weight regulation $[10,11]$. Melatonin ( $N$-acetyl-5-methoxytryptamine) in mammals is synthesized in several cells, tissues, and organs mainly for local utilization (autocrine and paracrine actions) and the circulating melatonin is mostly provided by the pineal gland $[11,12]$. It is known that melatonin is responsible for regulation of circadian rhythms, immune responses and mood, and reduces oxidative stress [13-16]. The roles of melatonin in obesity management have been studied in animal models of diet-induced obesity. These studies reported that melatonin might 
reduce weight, regulate energy expenditure, body fat mass, insulin secretion, and glucose/lipid metabolism [11,12,17-21]. Furthermore, mechanistic studies provide data, validating the powerful anti-inflammatory and antioxidant effects of melatonin [22]. Melatonin and its metabolites directly scavenge free radicals [15] and stimulate the activity and expression of antioxidant enzymes [23-25]. It also decreases production of proinflammatory cytokines by suppressing the mRNA expression of TNF- $\alpha$, IL-1 $\beta$, IL-6, and iNOS $[17,26,27]$. The overall findings indicate the efficacy of using melatonin as a therapeutic tool to prevent obesity-related disorders. To the best of authors' knowledge, there are no published reports related to the effect of melatonin on obesity complications in human subjects. The aim of this study was to evaluate the influence of melatonin supplementation on serum concentrations of inflammatory markers and the antioxidant status in obese females receiving weight control advice.

\section{Subjects and Methods}

In this randomized, double-blind, placebo-controlled trial, 46 volunteer healthy obese women who were referred to nutrition consultation in outpatient clinics during February 2013 and July 2013, were recruited. The inclusion criteria were body mass index $(\mathrm{BMI}) \geq 30 \mathrm{~kg} / \mathrm{m}^{2}$ and age $20-50$ years with conserved weight during the last 6 months. Exclusion criteria were pregnancy and lactation, menopause, smoking, alcohol consumption, having endocrine or kidney disease, depression, taking tranquilizers, contraceptives, anti-inflammatory agents, glucose and lipid- lowering drugs or taking any antioxidant supplements in the last 3 months. The research protocol was approved by Ethic Committee of Tabriz University of Medical Science (Ethic code: 924) and was registered in the Iranian Registry of Clinical Trials website (IRCT2012122411867N1). All subjects were made aware of the contents of the study, and a written informed consent document was obtained. The eligible participants were randomly allocated to intervention-placebo groups based on random block procedure produced by Random Allocation Software (RAS). Subjects and all who involved in enrolling participants, administering interventions, assessing outcomes, and analyzing data were blind to group assignments. Sample size was determined based on the information derived from the similar study [32]. The confidence level was set as $95 \%$ and formula $\mathrm{N}=\left[(\mathrm{Z1}-\alpha / 2+\mathrm{Z1}-\beta)^{2}\left(\mathrm{SD}_{1}{ }^{2}+\mathrm{SD}_{2}{ }^{2}\right)\right] / \Delta^{2}$ was used to calculate the 23 samples in each group. Along with a low-calorie diet, the melatonin treated group $(n=23)$, received $6 \mathrm{mg}$ melatonin (2 melatonin tablets, $3 \mathrm{mg}$ each; Nature Made, USA) once a day $2 \mathrm{~h}$ before bed time while control subjects $(n=23)$ received $6 \mathrm{mg}$ placebo, which were carefully matched in appearance with that of the melatonin tablets ( 2 tablets, $3 \mathrm{mg}$ each containing cellulose, silicon dioxide, and starch) once a day $2 \mathrm{~h}$ before bed time for 40 days. Total energy expenditure (TEE) was determined individually by calculating resting energy expenditure (REE) (using the Mifflin-St Jeor equation), multiplied by the estimated physical activity level (PAL) coefficient, which was then multiplied by 1.1 as thermic effect of food (TEF) coefficient by the dietitian. Low calorie diets were designed with a calorie deficit of $500 \mathrm{kcal} /$ day to achieve $0.5 \mathrm{~kg} /$ week weight loss in each obese woman. Subjects were monitored 3 times during the study for possible side effects of supplementation. Compliance was assessed by a tablet count. Subjects who consumed less than
$90 \%$ of the planned number of tablets were excluded from the study. Demographic and clinical data were obtained by interviewing the subjects.

\section{Anthropometric measurements}

At the onset and end of the study, body weight was recorded to nearest $0.1 \mathrm{~kg}$ with Seca scale and height was recorded to nearest $0.5 \mathrm{~cm}$ with a mounted tape. BMI was calculated as the weight in $\mathrm{kg}$ divided by the square of the height in meters. Obesity was defined as $\mathrm{BMI} \geq 30$ [1]. Waist and hip circumference was measured using a plasticized nonelastic measuring tape accurate to $0.5 \mathrm{~cm}$.

\section{Laboratory tests}

Before and after intervention, blood samples were collected after an overnight fasting of $12 \mathrm{~h}$ and serum samples were stored at $-70^{\circ} \mathrm{C}$ until biochemical analyses.

The serum levels of total antioxidant capacity (TAC) were measured using a Randox (Crumlin, County Antrim, United Kingdom) total antioxidant status kit in which 2,2-azino-bis(3-ethylbenzothiazoline-6-sulfonic acid) (ABTS) is incubated with a peroxidase and $\mathrm{H}_{2} \mathrm{O}_{2}$ to produce the radical cation ABTS+ $\bullet$. MDA levels were determined by the thiobarbituric acid reaction with acid, which was extracted with $n$-butanol, and measured spectrophotometrically at a wavelength of $523 \mathrm{~nm}$. Serum levels of TNF- $\alpha$ and IL-6 were measured using platinum enzyme-linked immunosorbent assay (ELISA) kits (DIA Source Immuno Assays S.A, Belgium) and were determined according to the instructions of each kit. Using an ELISA plate reader (Awareness, Model stat fax 2100, USA) at a wavelength of $450 \mathrm{~nm}$, the color changes were measured. Highsensitivity C-reactive protein assay (hsCRP) concentration was measured by latex-particle-enhanced immunoturbidometric assay.

\section{Statistical analysis}

Statistical analysis was performed using Statistical Package for the Social Sciences (SPSS, version16, Chicago, IL, USA). Normal distribution of data was verified with Kolmogorov-Smirnov test. A logarithmic transformation was used to normalize non-normally distributed variables. Mean values and standard deviation were calculated. The paired $t$-test was used to compare withingroup differences at baseline and after 40 days. After calculating percentage changes \{using the formula: [(after intervention values-baseline values)/baseline values $] \times 100$ \}, between groups comparisons were made by independent $t$-test. A p-value of $<0.05$ was considered statistically significant.

\section{Results}

$\nabla$

Forty-six individuals were recruited for the study. In the melatonin group, one subject consumed less than $90 \%$ of the planned number of tablets because of taking tranquilizers and was excluded from the study. In the placebo group, one subject withdrew because of failure to follow weight loss diet. Thus, 44 subjects (melatonin group $n=22$; placebo group $=22$ ) completed the study with the mean age of $33.86 \pm 6.94$ years in the melatonin group and $34.86 \pm 7.29$ years in the placebo group. No significant differences were noted in demographic characteristics $(\mathrm{p} \geq 0.05)$. Anthropometric data are presented in $\odot$ Table 1 . There were no significant differences in the baseline measures between the melatonin and placebo groups ( $p>0.05)$. Since all subjects were 


\begin{tabular}{|c|c|c|c|c|c|c|}
\hline & \multicolumn{2}{|c|}{ Melatonin group $(n=22)$} & \multicolumn{3}{|c|}{ Placebo group $(n=22)$} & \multirow[b]{2}{*}{$\mathbf{p}$} \\
\hline & Before & After & $\mathbf{p}$ & Before & After & \\
\hline Weight (kg) & $89.6 \pm 8.45$ & $87.1 \pm 9.15$ & 0.001 & $90.9 \pm 11.81$ & $88.6 \pm 11.48$ & 0.001 \\
\hline BMI $\left(\mathrm{kg} / \mathrm{m}^{2}\right)$ & $34.1 \pm 3.25$ & $33.1 \pm 3.47$ & 0.001 & $35.7 \pm 4.17$ & $34.8 \pm 4.09$ & 0.001 \\
\hline Waist circumference $(\mathrm{cm})$ & $94.9 \pm 6.6$ & $92.4 \pm 7.54$ & 0.001 & $97.3 \pm 8.26$ & $91.8 \pm 7.72$ & 0.001 \\
\hline Hip circumference (cm) & $117.9 \pm 7.63$ & $115.4 \pm 7.61$ & 0.001 & $119.1 \pm 9.96$ & $117.2 \pm 10.05$ & 0.002 \\
\hline
\end{tabular}

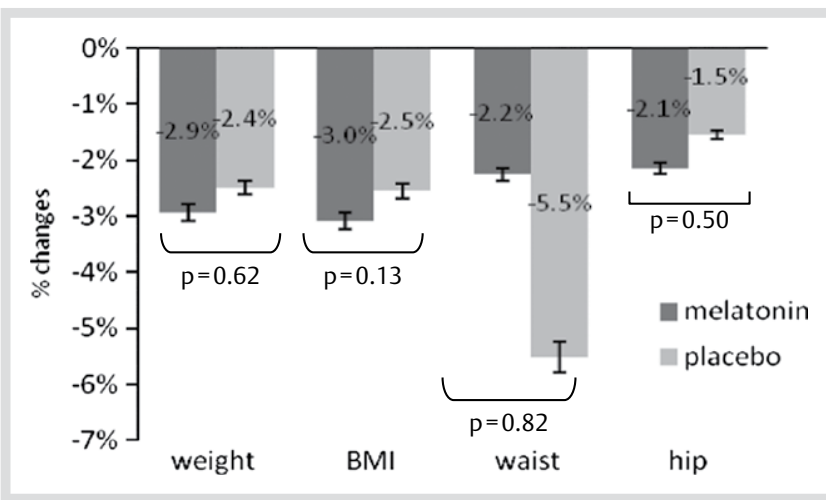

Fig. 1 Comparison of percentage changes of anthropometric measurements between the 2 study groups.

on a low calorie diet, subjects in both groups had significantly $(p<0.05)$ reduced weight, BMI, waist and hip circumference ( $\odot$ Table 1). As shown in $\odot$ Fig. 1 , the percentage changes of anthropometric indices between melatonin and placebo supplemented groups were not statistically significant $(p>0.05)$. Inflammatory and oxidative stress biomarkers are summarized in 8 Table 2. There were no significant differences in the baseline measures between the melatonin and placebo groups $(p>0.05)$. At the end of study, melatonin supplementation resulted in significant reduction in mean serum TNF- $\alpha$, IL- 6 , and hs-CRP whilst the placebo did not decrease those factors significantly. Furthermore, after intervention the mean MDA levels decreased in 2 groups; however, reduction level was significant only in melatonin group. While TAC level increased slightly in melatonin supplemented group, it decreased slightly in placebo group. As shown in $\odot$ Fig. 2, difference in percentage changes of inflammatory factors between 2 study groups were significant only for TNF- $\alpha(p=0.02)$ and IL-6 $(p=0.03)$. Comparison of percentage changes of oxidative stress biomarkers between the 2 study groups indicated no significant differences ( $\bullet$ Fig. 3 ).

\section{Discussion}

$\nabla$

Obesity, the major health problem, has become an epidemic disease throughout the world and is associated with numerous chronic disorders [1]. Nowadays, obesity management and its adverse health consequences require application of new generation of dietary supplements in addition to healthy lifestyles, which might help weight loss and ameliorate some detrimental effects of obesity $[8,9]$. Melatonin is one of the nutritional supplements, which has been taken into consideration for weight control lately [10-12]. Obesity induced lipo and glucotoxicity increases reactive oxygen species (ROS) production that triggers the proinflammatory cascade and potentiates tissue damage. Obesity is a state of chronic inflammation caused by altered pro- duction of proinflammatory markers (TNF- $\alpha$, IL-6, CRP) originates from white adipose tissue and increased oxidative damage $[2,3,5-7]$. During the past decade, several studies have supported the potential health benefits of melatonin including antioxidation, anti-inflammatory, immunomodulatory, body fat mass, and weight regulatory effects [10-13,24-28].

To the best of our knowledge, there are no published reports about the effect of melatonin supplementation in combination with a low calorie diet on anthropometric, inflammatory, and oxidative stress factors in obese women. Herein, we performed a study in healthy individuals with no major disease.

The results of the study by Rasmussen et al. [29] indicated that daily oral melatonin supplementation $(0.4-4 \mu \mathrm{g} / \mathrm{ml}$ for 12 weeks) in middle-aged rat significantly reduced body weight and visceral adiposity. Similar effects on body weight were observed in other experimental animal studies following melatonin supplementation $(4-10 \mathrm{mg} / \mathrm{kg}$ for $8-12$ weeks). In these studies melatonin significantly reduced body weight and other metabolic factors in rat models of high-fat diet-induced obesity $[19,30]$. In addition, long-term melatonin consumption $(4 \mathrm{mg} /$ $\mathrm{kg} /$ day for 16 weeks) in rats started before the establishment of obesity, attenuated weight gain and prevented the development of obesity induced metabolic alterations [21]. In type 2 diabetic patients, $5 \mathrm{mg} /$ day melatonin consumption for 30 days did not alter body weight and BMI [31]. In another clinical trail, $5 \mathrm{mg} /$ day melatonin supplementation for 2 months for patients with metabolic syndrome did not change the BMI [32]. Based on the current results, $6 \mathrm{mg} /$ day melatonin supplementation for 40 days in obese women received weight loss diet did not further reduce the body mass index (BMI), waist and hip circumferences since these alterations occurred in the placebo group who received low calorie diet ( $\bullet$ Table $\mathbf{1}, \otimes$ Fig. $\mathbf{1}$ ). It was expected that the melatonin-treated subjects would have had a further reduction in anthropometric parameters since other studies had showed melatonin to reduce body mass. In our study, subjects were treated with $6 \mathrm{mg}$ melatonin, which is a rather small dose in comparison with those used in animal studies $(4-10 \mathrm{mg} / \mathrm{kg})$. Higher doses of melatonin for longer treatment periods may result in further weight reduction.

In agreement with several studies, we did find beneficial effects of melatonin supplementation on antioxidant status. Melatonin supplementation significantly reduced serum malondialdehyde (MDA) levels ( $\bullet$ Table 2 ). The nonsignificant rise in TAC status of melatonin group might be due to short duration of intervention. Hussein et al. [17] reported that daily melatonin administration to obese rabbits ( $1 \mathrm{mg} / \mathrm{kg}$ subcutaneously for 4 weeks) significantly improved the TAC level. She et al. [30] noted that melatonin administration to obese rats $(4 \mathrm{mg} / \mathrm{kg}$ intraperitoneal injection for 8 weeks) significantly reduced MDA levels and elevated superoxide dismutase (SOD-1) activity. There are numerous studies documenting the ability of melatonin to reduce lipid peroxidation [33]. Melatonin supplementation in metabolic 


\begin{tabular}{|c|c|c|c|c|c|c|}
\hline & \multicolumn{2}{|c|}{ Melatonin group $(n=22)$} & \multicolumn{3}{|c|}{ Placebo group $(n=22)$} & \multirow[b]{2}{*}{$\mathbf{p}$} \\
\hline & Before & After & $\mathbf{p}$ & Before & After & \\
\hline hsCRP (mg/l) & $2.54 \pm 0.49$ & $1.67 \pm 0.27$ & 0.041 & $2.37 \pm 0.48$ & $1.44 \pm 0.21$ & 0.391 \\
\hline TNF- $\alpha(p g / m l)$ & $3.52 \pm 0.72$ & $1.73 \pm 0.07$ & 0.006 & $2.82 \pm 0.52$ & $2.01 \pm 0.38$ & 0.263 \\
\hline IL_6 (pg/ml) & $27.12 \pm 6.32$ & $16.34 \pm 6.32$ & 0.001 & $24.73 \pm 6.51$ & $21.11 \pm 5.94$ & 0.345 \\
\hline TAC (mmol/l) & $1.11 \pm 0.30$ & $1.14 \pm 0.45$ & 0.786 & $1.13 \pm 0.15$ & $1.08 \pm 0.21$ & 0.443 \\
\hline MDA (nmol/l) & $3.81 \pm 0.29$ & $2.79 \pm 0.29$ & 0.028 & $3.62 \pm 0.28$ & $2.96 \pm 0.37$ & 0.137 \\
\hline
\end{tabular}

Table 2 Inflammatory and oxidative stress parameters before and after intervention in the 2 study groups.

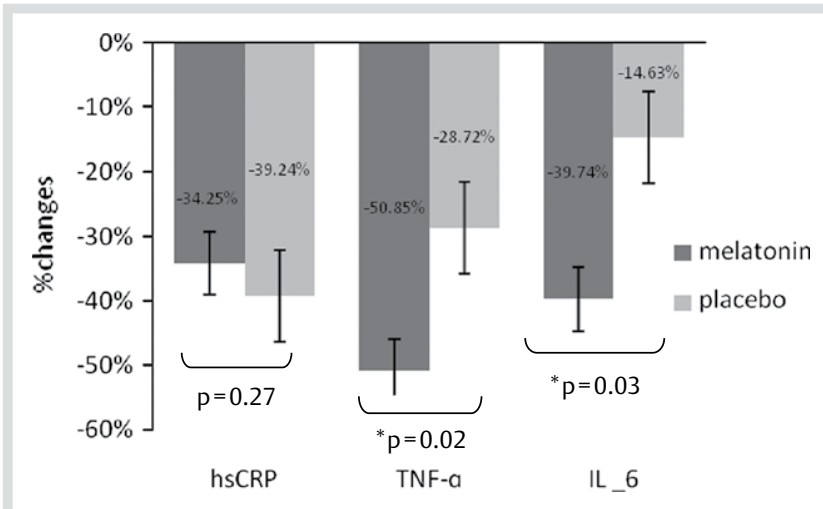

Fig. 2 Comparison of percentage changes of inflammatory parameters between the 2 study groups. ${ }^{*} p<0.05$, by independent samples $t$-test.

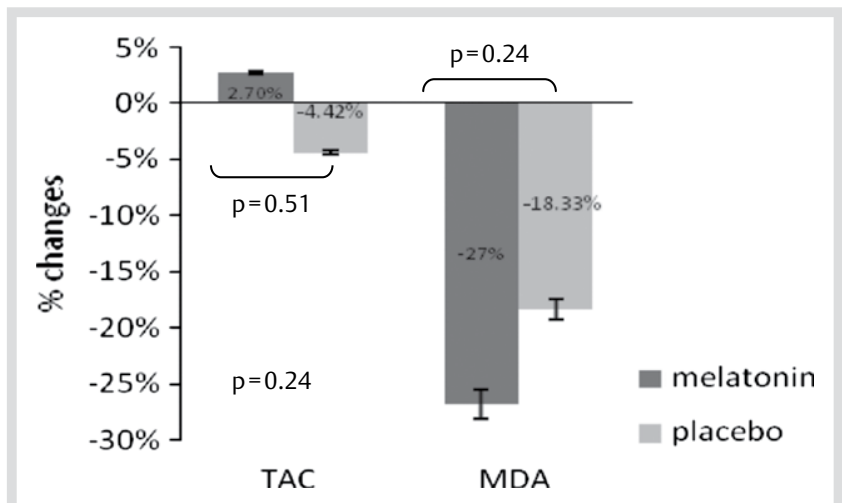

Fig. 3 Comparison of percentage changes of oxidative stress parameters between the 2 study groups.

syndrome patients ( $5 \mathrm{mg} /$ day) reduced MDA and increased catalase activity after 2 months [32]. Melatonin consumption $(5 \mathrm{mg} /$ day for 30 days) in type 2 diabetic patients resulted in a significant rise in SOD-1 activity and a reduction in the MDA level [31]. Moreover, $5 \mathrm{mg}$ /day melatonin supplementation for 30 days in primary essential hypertension patients significantly increased SOD-1 and catalase activity and reduced products of lipid peroxidation [34]. A very large body of evidences indicates that melatonin is a major scavenger of both oxygen-and nitrogenbased reactive molecules $[15,35]$. Melatonin enhances activity of several intracellular antioxidant enzymes, including SOD and glutathione peroxidase and stimulates glutathione production [28]. Furthermore, melatonin possesses genomic actions and regulates the expression of several genes, including those for SOD and glutathione peroxidase (GPx) [25]. Thus, melatonin influences both antioxidant enzyme activity and cellular mRNA levels of these detoxifying enzymes.
In the present study, supplementation of $6 \mathrm{mg} /$ day melatonin reduced inflammatory responses significantly in obese women through decreasing proinflammatory cytokines including TNF$\alpha$, IL-6, and hs-CRP values ( $\odot$ Table 2 ). The effect of melatonin on the suppression of proinflammatory cytokine production has been reported in earlier studies [22]. The in vivo study carried out by Jung et al. [36] showed that administration of melatonin $(50 \mathrm{mg} / \mathrm{kg}$ ) in rat inhibited the mRNA expression of TNF- $\alpha$, IL-6, IL-1 $\beta$, and iNOS. Veneroso et al. [37] also found that melatonin administration at a lower dose $(1 \mathrm{mg} / \mathrm{kg})$ in rats lowers mRNA levels of proinflammatory cytokines and protein level of inducible nitric oxide synthesase (iNOs) and cyclooxygenase-2 (COX2). In steatohepatitis patients, $5 \mathrm{mg} /$ day melatonin treatment for 1 month reduced TNF- $\alpha$ and IL- 6 levels significantly [38]. The mechanism of melatonin in the reduction of proinflammatory cytokines as well as iNOS production has been suggested via the inhibition of either expression or activation of nuclear factor-кB (NF-кB). Study limitations include the small sample size, which limits generalizability to a larger population, short duration of intervention, low melatonin dosage, and considering only female obese subjects.

\section{Conclusion}

$\nabla$

The results of the present study support the beneficial effects of melatonin supplementation for reducing obesity complications. Melatonin can be recommended as a part of comprehensive strategy involving diet and exercise in managing obesity. However, further studies with larger sample sizes, higher doses of melatonin, and longer intervention periods are needed to make concise conclusions.

\section{Authors' Note}

This article was written based on a data set of an M.Sc. thesis (Naimeh Mesri Alamdari) registered at Tabriz university of Medical Science.

\section{Author Contributions \\ $\nabla$}

All authors were involved in all parts of this study and approved the final manuscript. The contributions are as follows: Reza Mahdavi was responsible for supervising the study design as well as overseeing the study implementation. Naimeh Mesri Alamdari performed the study, collected, analyzed, interpreted all data, and drafted the manuscript. Neda Roshanravan collaborated in data collection. Neda Lotfi Yaghin and Elnaz Faramarzi collaborated in data analyzing and preparing the draft. Ali Reza 
Ostadrahimi participated in approving the final version of manuscript to be published.

\section{Acknowledgements}

The authors wish to thank Students Research Committee and Nutrition Research Center of Tabriz University of Medical Science for financial support. Grateful thanks go to all participants for their willingness to take part in the study.

\section{Conflict of Interest}

$\nabla$

The authors declare that they have no potential conflicts of interest with respect to the research, authorship, and/or publication of this article.

\section{References}

1 World health statistics [Internet]. WHO 2012; 110-111 Available from http://www.who.int

2 Lumeng $C N$, Saltiel AR. Inflammatory links between obesity and metabolic disease. J Clin Invest 2011; 121: 2111-2118

3 Mathieu P, Poirier P, Pibarot P, Lemieux I, Després JP. Visceral obesity the link among inflammation, hypertension, and cardiovascular disease. Hypertension 2009; 53: 577-584

4 McTiernan A. Obesity and cancer: the risks, science, and potential management strategies. Oncology 2005; 19: 871-881

5 Fantuzzi G. Adipose tissue, adipokines, and inflammation. J Allergy Clin Immunol 2005; 115: 911-919

6 Fernández-Sánchez A, Madrigal-Santillán E, Bautista M, Esquivel-Soto J, Morales-González Á, Esquivel-Chirino C, Durante-Montiel I, SánchezRivera G, Valadez-Vega C, Morales-González JA. Inflammation, oxidative stress, and obesity. Int J Mol Sci 2011; 12: 3117-3132

7 Beltowski J, Wojcicka G, Gorny D, Marciniak A. The effect of dietaryinduced obesity on lipid peroxidation, antioxidant enzymes and total plasma antioxidant capacity. J Physiol Pharmacol 2000; 51: 883-896

8 Wadden TA, Butryn ML, Wilson C. Lifestyle modification for the management of obesity. Gastroenterology 2007; 132: 2226-2238

9 Pillitteri JL, Shiffman S, Rohay JM, Harkins AM, Burton SL, Wadden TA. Use of dietary supplements for weight loss in the United States: results of a national survey. Obesity 2008; 16: 790-796

10 Jiménez-Aranda A, Fernández-Vázquez G, Campos D, Tassi M, VelascoPerez L, Tan DX, Reiter RJ, Agil A. Melatonin induces browning of inguinal white adipose tissue in Zucker diabetic fatty rats. J Pineal Res 2013; 55: 416-423

11 Tan DX, Manchester L, Fuentes-Broto L, Paredes S, Reiter R. Significance and application of melatonin in the regulation of brown adipose tissue metabolism: relation to human obesity. Obes Rev 2011; 12: 167-188

12 Cipolla-Neto J, Amaral F, Afeche S, Tan D, Reiter R. Melatonin, energy metabolism, and obesity. J Pineal Res 2014; 56: 371-381

13 Hardeland R, Madrid JA, Tan DX, Reiter RJ. Melatonin, the circadian multioscillator system and health: the need for detailed analyses of peripheral melatonin signaling. J Pineal Res 2012; 52: 139-166

14 Cardinali DP, Srinivasan V, Brzezinski A, Brown GM. Melatonin and its analogs in insomnia and depression. J Pineal Res 2012; 52: 365-375

15 Galano A, Tan DX, Reiter RJ. On the free radical scavenging activities of melatonin's metabolites, AFMK and AMK. J Pineal Res 2013; 54: 245-257

16 Calvo JR, González-Yanes C, Maldonado M. The role of melatonin in the cells of the innate immunity: a review. J Pineal Res 2013; 55: 103-120

17 Hussein MR, Ahmed OG, Hassan AF, Ahmed MA. Intake of melatonin is associated with amelioration of physiological changes, both metabolic and morphological pathologies associated with obesity: an animal model. Int J Exp Pathol 2006; 88: 19-29

18 Wolden-Hanson T, Mitton D, McCants R, Yellon S, Wilkinson C, Matsumoto A, Rasmussen $D$. Daily melatonin administration to middleaged male rats suppresses body weight, intraabdominal adiposity, and plasma leptin and insulin independent of food intake and total body fat. Endocrinology 2000; 141: 487-497
19 Prunet-Marcassus B, Desbazeille M, Bros A, Louche K, Delagrange P, Renard $P$, Casteilla L, Pénicaud L. Melatonin reduces body weight gain in Sprague Dawley rats with diet-induced obesity. Endocrinology 2003; 144: 5347-5352

20 Puchalski SS, Green JN, Rasmussen DD. Melatonin effect on rat body weight regulation in response to high-fat diet at middle age. Endocrine 2003; 21: 163-167

21 Nduhirabandi F, Du Toit EF, Blackhurst D, Marais D, Lochner A. Chronic melatonin consumption prevents obesity-related metabolic abnormalities and protects the heart against myocardial ischemia and reperfusion injury in a prediabetic model of diet-induced obesity. J Pineal Res 2011; 50: 171-182

22 Mauriz JL, Collado PS, Veneroso C, Reiter RJ, González-Gallego J. A review of the molecular aspects of melatonin's anti-inflammatory actions: recent insights and new perspectives. J Pineal Res 2013; 54: 1-14

23 Barlow-Walden L, Reiter R, Abe M, Pablos M, Menendez-Pelaez A, Chen $L-D$, Poeggeler $B$. Melatonin stimulates brain glutathione peroxidase activity. Neurochem Int 1995; 26: 497-502

24 Rodriguez C, Mayo JC, Sainz RM, Antolin I, Herrera F, Martin V, Reiter $R J$. Regulation of antioxidant enzymes: a significant role for melatonin. J Pineal Res 2004; 36: 1-9

25 Tomás-Zapico C, Coto-Montes A. A proposed mechanism to explain the stimulatory effect of melatonin on antioxidative enzymes. J Pineal Res 2005; 39: 99-104

26 Cuzzocrea S, Reiter RJ. Pharmacological actions of melatonin in acute and chronic inflammation. Curr Top Med Chem 2002; 2: 153-165

27 Radogna F, Diederich $M$, Ghibelli $L$. Melatonin: a pleiotropic molecule regulating inflammation. Biochem Pharmaco 2010; 80: 1844-1852

28 Reiter RJ, Tan D, Burkhardt S. Reactive oxygen and nitrogen species and cellular and organismal decline: amelioration with melatonin. Mech Ageing Dev 2002; 123: 1007-1019

29 Rasmussen DD, Boldt BM, Wilkinson C, Yellon SM, Matsumoto AM. Daily melatonin administration at middle age suppresses male rate visceral fat, plasma leptin, and plasma insulin to youthful levels. Endocrinology 1999; 140: 1009-1012

30 She $M$, Deng $X$, Guo Z, Laudon $M, H u Z$, Liao $D$, Hu $X$, Luo $Y$, Shen $Q$ $S u$ Z. NEU-P11, a novel melatonin agonist, inhibits weight gain and improves insulin sensitivity in high-fat/high-sucrose-fed rats. Pharmacol Res 2009; 59: 248-253

31 Kędziora-Kornatowska K, Szewczyk-Golec K, Kozakiewicz M, Pawluk H, Czuczejko J, Kornatowski T, Bartosz G, Kędziora J. Melatonin improves oxidative stress parameters measured in the blood of elderly type 2 diabetic patients. J Pineal Res 2009; 46: 333-337

32 Koziróg M, Poliwczak AR, Duchnowicz P, Koter-Michalak M, Sikora J, Broncel $M$. Melatonin treatment improves blood pressure, lipid profile, and parameters of oxidative stress in patients with metabolic syndrome. J Pineal Res 2011; 50: 261-266

33 Reiter RJ, Paredes SD, Manchester LC, Tan D-X. Reducing oxidative/ nitrosative stress: a newly-discovered genre for melatonin. Crit Rev Biochem Mol Biol 2009; 44: 175-200

34 Kędziora-Kornatowska K, Szewczyk-Golec K, Czuczejko J, Pawluk H, Van Marke de Lumen K, Kozakiewicz M, Bartosz G, Kędziora J. Antioxidative effects of melatonin administration in elderly primary essential hypertension patients. J Pineal Res 2008; 45: 312-317

35 Tan D-X, Chen L, Poeggeler B, Manchester L, Reiter R. Melatonin: a potent, endogenous hydroxyl radical scavenger. Endocr J 1993; 1: 57-60

36 Jung KH, Hong SW, Zheng HM, Lee HS, Lee H, Lee DH, Lee SY, Hong SS. Melatonin ameliorates cerulein-induced pancreatitis by the modulation of nuclear erythroid 2-related factor 2 and nuclear factor-kappaB in rats. J Pineal Res 2010; 48: 239-250

37 Veneroso C, Tuñón MJ, González-Gallego J, Collado PS. Melatonin reduces cardiac inflammatory injury induced by acute exercise. J Pineal Res 2009; 47: 184-191

38 Cichoz-Lach H, Celinski K, Konturek P, Konturek S, Slomka M. The effects of L-tryptophan and melatonin on selected biochemical parameters in patients with steatohepatitis. J Physiol Pharmacol 2010; 61: 577-580 